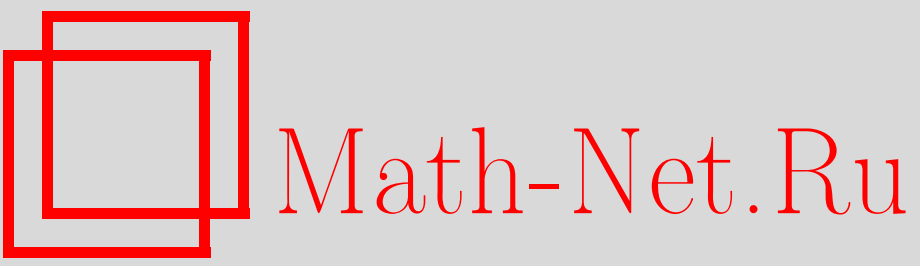

Ал. Б. Замолодчиков, Трехточечная функция минимальной лиувиллевской гравитации, ТМФ, 2005, том 142, номер 2, 218-234

DOI: https://doi.org/10.4213/tmf1777

Использование Общероссийского математического портала Math-Net.Ru подразумевает, что вы прочитали и согласны с пользовательским соглашением

http://www.mathnet.ru/rus/agreement

Параметры загрузки:

IP : 54.197 .130 .99

26 апреля 2023 г., $17: 44: 47$ 
ТЕОРЕТИЧЕСКАЯ

И МАТЕМАТИЧЕСКАЯ

ФИЗИКА

Том 142, № 2

февраль, 2005

(C) 2005 г.

Ал. Б. Замолодчиков*

\title{
ТРЕХТОЧЕЧНАЯ ФУНКЦИЯ МИНИМАЛЬНОЙ ЛИУВИЛЛЕВСКОЙ ГРАВИТАЦИИ
}

\begin{abstract}
Заново рассматривается задача о структурных константах операторных произведений в минимальных моделях конфформной теории поля. Воспроизводятся известные ранее константы и дается их представление в виде, удобном, в частности, для применения в лиувиллевской гравитации. Обсуждается аналитическое соотношение между полученными выражениями и структурными константами в теории поля Лиувилля. Приводится также общий вид трех- и двухточечных корреляционных чисел на сфере в минимальной лиувиллевской гравитации.
\end{abstract}

Ключевые слова: конформная теория поля, лиувиллевская гравитация, минимальные модели.

\section{1. ЛИУВИЛЛЕВСКАЯ ГРАВИТАЦИЯ}

Под гравитацией обычно понимается динамическая теория метрической структуры на некотором многообразии. В размерности 2 в качестве последнего берется двумерная поверхность $\Sigma$ (компактная или некомпактная) с некоторой топологией, снабженная римановой метрикой $g_{a b}(x)$. Чтобы избежать проблемы модулей, мы в данной работе будем всегда предполагать, что $\Sigma$ - сфера. Будем рассматривать также евклидов вариант гравитации, т.е. предполагать, что $g_{a b}$ невырожденна и с положительной сигнатурой. В подходе, основанном на интеграле по траекториям, задача сводится к вычислению функционального интеграла по всем римановым метрикам $D[g]$, рассматриваемым по модулю эквивалентности при диффеоморфизмах. Например, гравитационная статистическая сумма сферы формально записывается как

$$
Z=\int D[g] e^{-A_{\mathrm{eff}}[g]} .
$$

Здесь предполагается, что $A_{\text {eff }}[g]$ - эффективное действие, индуцированное неким общековариантным "материальным" полем теории на поверхности. Общая ковариантность обеспечивает инвариантность $A_{\text {eff }}[g]$ относительно диффеоморфизмов.

* Laboratoire de Physique Mathématique, Université Montpellier II, Montpellier, France; Институт теоретической и экспериментальной физики, Москва, Россия.

E-mail: zamolod@lpm.univ-montp2.fr 
В обшем случае массивной материи $A_{\text {eff }}[g]$ является нелокальным и весьма сложным функционалом метрики. Задача (1) представляется очень сложной. Радикальное упрошение возникает, однако, если вся материя, индуцирующая $A_{\mathrm{eff}}[g]$, является "критической”, т.е. описывается конформной теорией поля (КТП). Тогда форма $A_{\text {eff }}[g]$ является весьма универсальной и простой и называется действием Лиувилля. Этот факт был впервые обнаружен Поляковым в 1981 г. [1] путем прямых вычислений со свободными полями. В обшей КТП это утверждение просто следует из формы конформной аномалии. Более того, концептуально этот вид эффективного действия можно принять (при некоторых дополнительных допушениях) за само определение КТП.

Из-за инвариантности $A_{\text {eff }}[g]$ относительно диффеоморфизмов возникает задача фиксации калибровки в (1). Одна из наиболее удобных возможностей состоит в выборе конформной калибровки, где координаты на $\Sigma$ (локально) выбираются так, что

$$
g_{a b}(x)=e^{2 b \phi(x)} \delta_{a b}
$$

(это всегда возможно в размерности 2). Входящий сюда масштабный множитель описывает квантовое поле $\phi(x)$, называемое полем Лиувилля (определение параметра $b$ см. ниже). Можно также фиксировать калибровку ковариантным образом, выбирая произвольную метрику $g_{a b}^{(0)}(x)$ в качестве опорной и требуя, чтобы выполнялось соотношение

$$
g_{a b}(x)=e^{2 b \phi(x)} g_{a b}^{(0)}(x)
$$

В последнем подходе поле Лиувилля $\phi$ является обычным скаляром при координатных преобразованиях. Калибровка (2) тогда означает специальный выбор системы координат и выбор $g_{a b}^{(0)}(x)=\delta_{a b}$ в этих координатах.

Как обычно, фиксация калибровки приводит к появлению детерминанта ФаддееваПопова. В нашем случае его можно описать $B C$-системой спина $(2,-1)$ :

$$
A_{\mathrm{gh}}=\frac{1}{\pi} \int(C \bar{\partial} B+\bar{C} \partial \bar{B}) d^{2} x
$$

Эта система также представляет собой КТП с центральным зарядом $c_{\mathrm{gh}}=-26$, а потому детерминант, связанный с фиксацией калибровки, снова сводится к действию Лиувилля. Этот факт также был замечен в работе [1]. Гравитационная статистическая сумма принимает вид

$$
Z_{\mathrm{g}}=\int D[\phi] e^{-A_{\mathrm{L}}[\phi]},
$$

где $A_{\mathrm{L}}[\phi]$ - действие Лиувилля, индуцированное полями материи и гостов.

Возникает некоторая проблема с мерой интегрирования $D[\phi]$ по конфигурациям поля Лиувилля [1]. Полное определение интеграла по траекториям (5) требует ультрафиолетового обрезания, которое с физической точки зрения само должно зависеть от масштабного множителя $e^{2 b \phi}$. Это означает, что мера интегрирования отличается от 
обычной (линейной) меры интегрирования, в которой обрезание определяется относительно некоторой фиксированной метрики. Прямое вычисление интеграла (5) с этой нелинейной мерой оказывается весьма сложным и технически, и концептуально. Однако в работах [2] было предложено свести эффект этой сложной нелинейной меры к некоторой конечной перенормировке параметров. Это означает, что обыкновенную линейную меру (по отношению к фиксированной опорной метрике) можно согласованным образом использовать в $(5)$, если параметры в $A_{\mathrm{L}}[\phi]$ выбраны подходящим образом. Тогда перенормированные параметры можно определить из условий согласованности. У этого предположения нет действительно хорошего теоретического обоснования. Единственные серьезные аргументы в его пользу могут появиться из реальных вычислений в его рамках и сравнения результатов с другими известными фактами в двумерной квантовой гравитации. К последним относятся результаты дискретного, или матричного, подхода (см., например, обзоры [3] и приведенную там библиографию) и теоретико-полевые вычисления в другой калибровке (называемой калибровкой светового конуса, или калибровкой Полякова) [4].

После выполнения этой процедуры перенормированное действие Лиувилля принимает вид

$$
A_{\mathrm{L}}[\phi]=\int\left(\frac{1}{4 \pi}\left(\partial_{a} \phi\right)^{2}+\mu e^{2 b \phi}\right) d^{2} x,
$$

где $\mu$ - еше один параметр, называемый космологической постоянной. Действие (6) также описывает КТП с центральным зарядом

$$
c_{\mathrm{L}}=1+6 Q^{2}
$$

где “фоновый заряд” $Q$ связан с параметром $b$ как

$$
Q=b+b^{-1}
$$

Упомянутое выше условие согласованности, предложенное Давидом, а также Дистлером и Каваи, фиксирует параметр $b$, исходя из условия, что полный центральный заряд объединенных КТП-материи, поля Лиувилля и гостов,

$$
A_{\mathrm{g}}=A_{\mathrm{CFT}}+A_{\mathrm{L}}+A_{\mathrm{gh}}
$$

обращается в нуль (что в действительности означает независимость физических наблюдаемых от выбора опорной метрики в (3)):

$$
c_{\mathrm{M}}+c_{\mathrm{L}}=26
$$

Здесь $c_{\mathrm{M}}$ - центральньй заряд КТП-материи, а $A_{\mathrm{CFT}}$ обозначает ее формальное действие.

Действие (9) означает, что в критической гравитации три теории поля (конформной материи, поля Лиувилля $\phi$ и гостов) формально не взаимодействуют друг с другом, за 
исключением взаимодействия через конформную аномалию. В корреляционных функциях всякое примарное поле материи $\Phi$ размерности $\Delta$ должно быть самосогласованным образом "одето" подходящей экспонентой поля Лиувилля $e^{2 a \phi}$ так, чтобы образовать составное поле размерности $(1,1)$ :

$$
O=\Phi e^{2 a \phi}
$$

В теории поля Лиувилля (6) экспонента $e^{2 a \phi}$ имеет размерность

$$
\Delta_{a}=a(Q-a)
$$

так что параметр одевания $a$ определяется из условия

$$
\Delta+\Delta_{a}=1
$$

Корреляционные функции одетых операторов распадаются (до интегрирования по модулям) в произведение корреляционных функций Лиувилля и материи:

$$
\left\langle O_{1}\left(x_{1}\right) \ldots O_{n}\left(x_{n}\right)\right\rangle_{\mathrm{LG}}=\left\langle\Phi_{1}\left(x_{1}\right) \ldots \Phi_{n}\left(x_{n}\right)\right\rangle_{\mathrm{CFT}}\left\langle e^{2 a_{1} \phi}\left(x_{1}\right) \ldots e^{2 a_{n} \phi}\left(x_{n}\right)\right\rangle_{\mathrm{L}}
$$

где $\langle\ldots\rangle_{\mathrm{LG}},\langle\ldots\rangle_{\mathrm{CFT}}$ и $\langle\ldots\rangle_{\mathrm{L}}-$ корреляционные функции в теории лиувиллевской гравитации, КТП-материи и теории Лиувилля, соответственно.

Хорошо известно, что на сфере из-за сушествования избыточных конформных векторных полей Киллинга конформная калибровка требует некоторой дополнительной фиксации. Чтобы получить ее, необходимо снабдить три произвольных поля в корреляционной функции (14) гостовскими множителями $C \bar{C}$, которые придадут произведению полную размерность $(0,0)$. Остальные вставки имеют размерность $(1,1)$ и могут быть проинтегрированы по своим координатам. Получаюшаяся таким образом корреляционная функция

$$
\begin{aligned}
\left\langle O_{1} \ldots O_{n}\right\rangle_{\mathrm{G}}= & \int\left\langle C \bar{C}\left(x_{1}\right) \ldots C C\left(x_{3}\right)\right\rangle_{\mathrm{gh}}\left\langle\Phi_{1}\left(x_{1}\right) \ldots \Phi_{n}\left(x_{n}\right)\right\rangle_{\mathrm{CFT}} \times \\
& \times\left\langle e^{2 a_{1} \phi}\left(x_{1}\right) \ldots e^{2 a_{n} \phi}\left(x_{n}\right)\right\rangle_{\mathrm{L}} d^{2} x_{4} \ldots d^{2} x_{n}
\end{aligned}
$$

более не зависит от координат (а потому и от выбора калибровки) и скорее заслуживает названия корреляционного числа. Мы будем использовать для этих чисел обозначение $\langle\ldots\rangle_{\mathrm{G}}$. Эти калибровочно-инвариантные корреляционные числа - один из наиболее интересных объектов в двумерной гравитации. Например, предположим, что рассматривается более сложная гравитация, где материя на поверхности не чисто критическая, а описывается некоторым возмушением КТП:

$$
A_{\mathrm{M}}=A_{\mathrm{CFT}}+\lambda \int \Phi(x) d^{2} x
$$


Здесь $\Phi$ - релевантное примарное поле материи (размерности $\Delta$ ), а $\lambda$ - соответствующая константа взаимодействия. В гравитационном окружении поле $\Phi$ одевается полем $e^{2 a \phi}$ (где $a$ определено в (12) и (13)). Пертурбативное разложение по $\lambda$

$$
Z(\lambda, \mu)=Z(0, \mu) \sum_{n=0}^{\infty} \frac{(-\lambda)^{n}}{n !} a_{n}
$$

где

$$
\begin{aligned}
a_{0}= & 1 \\
a_{n}= & \int\left\langle C \bar{C}\left(x_{1}\right) \ldots C C\left(x_{3}\right)\right\rangle_{\mathrm{gh}}\left\langle\Phi\left(x_{1}\right) \ldots \Phi\left(x_{n}\right)\right\rangle_{\mathrm{CFT}} \times \\
& \times\left\langle e^{2 a \phi}\left(x_{1}\right) \ldots e^{2 a \phi}\left(x_{n}\right)\right\rangle_{\mathrm{L}} d^{2} x_{4} \ldots d^{2} x_{n}, \quad n \geqslant 3,
\end{aligned}
$$

таким образом выражается через многоточечные корреляционные числа (15) (случаи $n=1$ и $n=2$ несколько более специальные и будут рассмотрены ниже).

В данной статье мы интересуемся специальным случаем двумерной индуцированной критической гравитации - минимальной гравитацией (МГ). Это название означает, что материя описыватся только одной КТП-минимальной моделью $\mathcal{M}_{p, p^{\prime}}[5]$, где $\left(p, p^{\prime}\right)$-пара взаимно простых целых чисел. Результаты дискретного подхода к двумерной гравитации [3] в большой степени указывают на то, что МГ является точно решаемой. Например, скейлинговые функции, подобные (17), а потому и корреляционные числа (15) могут быть найдены в рамках матричных моделей явно. Однако выявление потенциальных возможностей лиувиллевской гравитации путем воспроизведения точных результатов, полученных дискретными методами, все еше остается важнейшей нерешенной задачей.

Настоящая работа посвяшена простейшим корреляционным числам в МГ - трехточечным. В этом случае в уравнении (15) не надо проводить интегрирования, и наша задача состоит просто в умножении трехточечной функции Лиувилля на трехточечную функцию в теории $\mathcal{M}_{p, p^{\prime}}$. Эта несложная работа выполнена в следующих разделах, где также приводятся замечания об аналитическом соотношении между минимальными моделями и теорией поля Лиувилля.

\section{2. ТРЕХТОЧЕЧНАЯ ФУНКЦИЯ ЛИУВИЛЛЯ}

Трехточечная функция экспоненциального поля $e^{2 a \phi}$

$$
\begin{aligned}
\left\langle e^{2 a_{1} \phi}\left(x_{1}\right) e^{2 a_{2} \phi}\left(x_{2}\right) e^{2 a_{3} \phi}\left(x_{3}\right)\right\rangle_{\mathrm{L}}= & \\
= & \frac{C_{\mathrm{L}}\left(a_{1}, a_{2}, a_{3}\right)}{\left(x_{12} \bar{x}_{12}\right)^{\Delta_{1}+\Delta_{2}-\Delta_{3}}\left(x_{23} \bar{x}_{23}\right)^{\Delta_{2}+\Delta_{3}-\Delta_{1}}\left(x_{31} \bar{x}_{31}\right)^{\Delta_{3}+\Delta_{1}-\Delta_{2}}}
\end{aligned}
$$

в теории поля Лиувилля была открыта Дорном и Отто [6] в 1992 г. Координатная зависимость (19) включает размерность $\Delta_{i}=\Delta_{a_{i}}$ экспоненциального поля, заданную равенством (12). Эта зависимость является стандартной, и поэтому мы опустим данный 
множитель и будем называть трехточечной функцией $C_{\mathrm{L}}\left(a_{1}, a_{2}, a_{3}\right)$. В обозначениях из работы [7] она имеет явный вид

$$
\begin{aligned}
& C_{\mathrm{L}}\left(a_{1}, a_{2}, a_{3}\right)=\left(\pi \mu \gamma\left(b^{2}\right) b^{2-2 b^{2}}\right)^{\left(Q-a_{1}-a_{2}-a_{3}\right) / b} \times \\
& \quad \times \frac{\Upsilon(b) \Upsilon\left(2 a_{1}\right) \Upsilon\left(2 a_{2}\right) \Upsilon\left(2 a_{3}\right)}{\Upsilon\left(a_{1}+a_{2}+a_{3}-Q\right) \Upsilon\left(a_{1}+a_{2}-a_{3}\right) \Upsilon\left(a_{2}+a_{3}-a_{1}\right) \Upsilon\left(a_{3}+a_{1}-a_{2}\right)} .
\end{aligned}
$$

Здесь $\Upsilon(x)=\Upsilon_{b}(x)$ - специальная функция, связанная с двойной гамма-функцией Барнса [8] (точные определения и свойства см. в [7]). Здесь следует заметить, что (20) ненормализованная корреляционная функция. Для использования в разложениях вида (17) ее следует разделить на статистическую сумму Лиувилля для сферы. Ниже мы приводим замечания по этому поводу.

Позднее, в 1995 г., выражение (20) было воспроизведено Тешнером [9] более систематическим способом с помошью техники конформного бутстрапа. Оказывается, что самосогласованность (бутстрапа) приводит к требованию, чтобы $C_{\mathrm{L}}\left(a_{1}, a_{2}, a_{3}\right)$ удовлетворяли функциональным соотношениям

$$
\begin{aligned}
& \frac{C_{\mathrm{L}}\left(a_{1}+b, a_{2}, a_{3}\right)}{C_{\mathrm{L}}\left(a_{1}, a_{2}, a_{3}\right)}=-\frac{\gamma\left(-b^{2}\right)}{\pi \mu} \times \\
& \quad \times \frac{\gamma\left(2 a_{1} b+b^{2}\right) \gamma\left(2 a_{1} b\right) \gamma\left(b\left(a_{2}+a_{3}-a_{1}\right)-b^{2}\right)}{\gamma\left(b\left(a_{1}+a_{2}+a_{3}\right)-1-b^{2}\right) \gamma\left(b\left(a_{1}+a_{2}-a_{3}\right)\right) \gamma\left(b\left(a_{1}+a_{3}-a_{2}\right)\right)}, \\
& \frac{C_{\mathrm{L}}\left(a_{1}+b^{-1}, a_{2}, a_{3}\right)}{C_{\mathrm{L}}\left(a_{1}, a_{2}, a_{3}\right)}=-\frac{\gamma\left(-b^{-2}\right)}{\pi \tilde{\mu}} \times \\
& \quad \times \frac{\gamma\left(2 a_{1} b^{-1}+b^{-2}\right) \gamma\left(2 a_{1} b^{-1}\right) \gamma\left(b^{-1}\left(a_{2}+a_{3}-a_{1}\right)-b^{-2}\right)}{\gamma\left(b^{-1}\left(a_{1}+a_{2}+a_{3}\right)-1-b^{-2}\right) \gamma\left(b^{-1}\left(a_{1}+a_{2}-a_{3}\right)\right) \gamma\left(b^{-1}\left(a_{1}+a_{3}-a_{2}\right)\right)} .
\end{aligned}
$$

Здесь $\tilde{\mu}$ - “дуальная космологическая постоянная", связанная с $\mu$ соотношением (см. [7])

$$
\left(\tilde{\mu} \gamma\left(b^{-2}\right)\right)^{b}=\left(\mu \gamma\left(b^{2}\right)\right)^{1 / b} .
$$

В обшем случае $b$ и $b^{-1}$ несоизмеримы и функция (20) является единственным решением этой системы.

Выражение (20) предполагает специальную нормировку лиувиллевских экспоненциальных полей. Она фиксируется двухточечной функцией

$$
\left\langle e^{2 a \phi}(x) e^{2 a \phi}(0)\right\rangle_{\mathrm{L}}=\frac{D_{\mathrm{L}}(a)}{(x \bar{x})^{2 \Delta_{a}}},
$$

где

$$
D_{\mathrm{L}}(a)=\frac{\left(\pi \mu \gamma\left(b^{2}\right)\right)^{(Q-2 a) / b}}{b^{2}} \frac{\gamma\left(2 a b-b^{2}\right)}{\gamma\left(2-2 a b^{-1}+b^{-2}\right)} .
$$

Это снова ненормированное выражение. Как использовать его в двухточечном корреляционом числе, также обсуждается ниже. 


\section{3. ОБОБЩЕННЫЕ МИНИМАЛЬНЫЕ МОДЕЛИ}

Минимальная модель КТП $\mathcal{M}_{p, p^{\prime}}$ характеризуется центральным зарядом

$$
c_{\mathrm{M}}=1-6\left(\beta^{-1}-\beta\right)^{2},
$$

где

$$
\beta=\sqrt{\frac{p}{p^{\prime}}} .
$$

Ниже мы будем считать, что $p<p^{\prime}$, а потому $\beta<1$; удобно также ввести параметр

$$
q=\beta^{-1}-\beta
$$

Таким образом [5],

$$
c_{p / p^{\prime}}=1-6 q^{2}=1-\frac{6\left(p-p^{\prime}\right)^{2}}{p p^{\prime}} .
$$

Главная (унитарная) серия соответствует $p^{\prime}=p+1$.

В минимальных моделях предполагается, что параметр $\beta^{2}$ - рациональное число. Однако в данной работе мы будем стремиться к максимально возможной аналитичности. Поэтому попытаемся рассматривать $\beta$ как непрерывный вешественный (и позднее даже комплексньй) параметр до тех пор, пока это не будет приводить к явным проблемам. Соответствуюшее непрерывное семейство (формальных) моделей КТП называется обобщенной минимальной моделью $(\mathrm{OMM})$ и обозначается как $\mathcal{M}_{\beta}$.

Состав примарных операторов в минимальной модели дается множеством вырожденных полей $\Phi_{m, n}$, где $(m, n)$ - пара целых чисел. Их размерности выражаются формулой Каца [10]

$$
\Delta_{m, n}=\frac{\left(m \beta^{-1}-n \beta\right)^{2}-\left(\beta^{-1}-\beta\right)^{2}}{4}=\alpha_{m, n}\left(\alpha_{m, n}-q\right) .
$$

В такой параметризации имеются две возможности выбрать $\alpha_{m, n}$, различаюшиеся заменой $\alpha_{m, n} \leftrightarrow q-\alpha_{m, n}$. Для определенности выберем

$$
\alpha_{m, n}=\frac{(n-1) \beta}{2}-\frac{(m-1) \beta^{-1}}{2} .
$$

Все представления алгебры Вирасоро с центральным зарядом (25) и размерностью (29) вырожденны и имеют нуль-вектор на уровне $m n$. В минимальных моделях требуется обращение в нуль этих нуль-векторов. Это одно из основных требований в конструкции минимальных моделей [5]. Их следствием являются некие линейные дифференциальные уравнения для вырожденных примарных полей. Например, простейшие нетривиальные поля $\Phi_{1,2}$ и $\Phi_{2,1}$ удовлетворяют равенствам

$$
\begin{aligned}
\left(\partial^{2}-\beta^{2} T\right) \Phi_{1,2} & =0 \\
\left(\partial^{2} \Phi_{21}-\beta^{-2} T\right) \Phi_{2,1} & =0
\end{aligned}
$$


и аналогичным “левым" уравнениям, в которых $\partial$ заменено на $\bar{\partial}$ и правая компонента тензора натяжений $T=T_{z z}$ заменена левой $\bar{T}=T_{\bar{z} \bar{z}}$ (см. более подробный анализ в работе [5]). Эти дифференциальные уравнения ограничивают возможный вид операторного произведения вырожденных полей $\Phi_{m, n}$ до очень специального [5]:

$$
\Phi_{m_{1}, n_{1}} \Phi_{m_{2}, n_{2}}=\sum_{\left(m_{3}, n_{3}\right)} C_{\left(m_{1}, n_{1}\right)\left(m_{2}, n_{2}\right)}^{\left(m_{3}, n_{3}\right)}\left[\Phi_{m_{3}, n_{3}}\right]
$$

(мы опускаем здесь стандартные зависяшие от $x$ множители и обозначаем через [ $\left.\Phi_{m, n}\right]$ вклад примарного поля $\Phi_{m, n}$ и всех его конформных потомков).

Разумеется, структурные константы $C_{\left(m_{1}, n_{1}\right)\left(m_{2}, n_{2}\right)}^{\left(m_{3}, n_{3}\right)}$ зависят от нормировки полей $\Phi_{m, n}$. В данной работе мы примем стандартную нормировку КТП через двухточечные функции:

$$
\left\langle\Phi_{m, n}(x) \Phi_{m, n}(0)\right\rangle_{\mathrm{CFT}}=\frac{1}{(x \bar{x})^{2 \Delta_{m, n}}} .
$$

В этой нормировке структурные константы совпадают с трехточечными функциями:

$$
C_{\left(m_{1}, n_{1}\right)\left(m_{2}, n_{2}\right)}^{\left(m_{3}, n_{3}\right)}=C_{\left(m_{1}, n_{1}\right)\left(m_{2}, n_{2}\right)\left(m_{3}, n_{3}\right)}=\left\langle\Phi_{m_{1}, n_{1}} \Phi_{m_{2}, n_{2}} \Phi_{m_{3}, n_{3}}\right\rangle_{\mathrm{CFT}}
$$

(мы снова опускаем стандартные зависящие от $x$ множители).

Структурные константы в ОММ подчинены дальнейшим ограничениям, происходящим из правил слияния (fusion rules), которые также следуют из обращения нуль-векторов в нуль. Правила слияния по отдельности ограничивают возможные тройки индексов $\left(m_{1}, m_{2}, m_{3}\right)$ и $\left(n_{1}, n_{2}, n_{3}\right)$ в структурных константах. В действительности они эквивалентны алгебре слияния регулярных представлений алгебры $S L(2)$. Таким образом, структурные константы ОММ пропорциональны структурным константам алгебры слияния:

$$
C_{\left(m_{1}, n_{1}\right)\left(m_{2}, n_{2}\right)\left(m_{3}, n_{3}\right)} \sim f_{m_{1}, m_{2}, m_{3}} f_{n_{1}, n_{2}, n_{3}}
$$

где

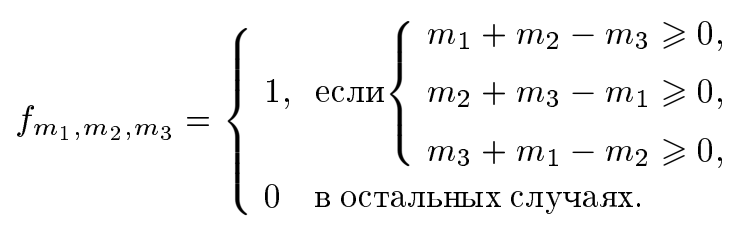

Структурные константы OMM $C_{\left(m_{1}, n_{1}\right)\left(m_{2}, n_{2}\right)\left(m_{3}, n_{3}\right)}$ были явно вычислены в виде некоторых произведений гамма-функций в работе Доценко и Фатеева [11]. В следующем разделе мы попытаемся получить их результат несколько иным способом, в большей степени отвечаюшим нашему намерению искать аналитичность. Для этого введем вместо дискретного набора вырожденных операторов $\Phi_{m, n}$ формальное семейство полей $\Phi_{\alpha}$, параметризованное непрерывным параметром $\alpha$. Они имеют размерности

$$
\Delta_{\alpha}=\alpha(\alpha-q)
$$


Далее используем технику бутстрапа для определения формальной трехточечной функции (снова с опущенной координатной зависимостью)

$$
C_{\mathrm{M}}\left(\alpha_{1}, \alpha_{2}, \alpha_{3}\right)=\left\langle\Phi_{\alpha_{1}} \Phi_{\alpha_{2}} \Phi_{\alpha_{3}}\right\rangle_{\mathrm{M}}
$$

как непрерывной функции трех ее параметров, причем такой, чтобы при выборе специальных значений (30) $\alpha_{i}=\alpha_{m_{i}, n_{i}}$ воспроизводились бы известные выражения Доценко-Фатеева.

Преж де чем обратиться к этой задаче, уместно сделать три замечания.

1. В настоящей минимальной модели $\mathcal{M}_{p, p^{\prime}}$ имеется конечное подмножество вырожденных примарных полей $\Phi_{m, n}$, для которых $1 \leqslant m \leqslant p-1$ и $1 \leqslant n \leqslant p^{\prime}-1$, замкнутое по отношению к операторному произведению (поля $\Phi_{m, n}$ и $\Phi_{p-m, p^{\prime}-n}$ отождествляются, вследствие чего конечное подмножество насчитывает всего $(p-1)\left(p^{\prime}-1\right) / 2$ полей) [5]. Поэтому в настояших минимальных моделях возможна самосогласованная редукция операторного состава модели $\mathcal{M}_{\beta}$ к этому конечному подмножеству примарных полей, что дает рациональную КТП. В ОММ при иррациональных $\beta^{2}$ такая редукция невозможна, и мы вынуждены рассматривать весь набор $\Phi_{m, n}$ с произвольными положительными целыми $(m, n)$. Остается неясным, согласована ли такая бесконечная алгебра с обшими требованиями квантовой теории поля. В частности, конструкция модулярно-инвариантной статистической суммы в ОММ, очевидно, встречается с серьезными сложностями. В настояшей работе мы предпочитаем занимать формальную позицию и забываем на время об этих важных вопросах.

2. Как будет видно в дальнейшем, непрерывная функция $C_{\mathrm{M}}\left(\alpha_{1}, \alpha_{2}, \alpha_{3}\right)$ (которая также будет построена ниже) при вырожденных значениях $\alpha_{i}=\alpha_{m_{i}, n_{i}}$ не всегда обрашается в нуль автоматически, если правила слияния (36) нарушаются. Иногда она дает некие конечные числа, интерпретация которых остается пока загадочной. Поэтому для получения правильного набора структурных констант в ОММ следует учитывать правила слияния по отдельности. Соотношение между $C_{\left(m_{1}, n_{1}\right)\left(m_{2}, n_{2}\right)\left(m_{3}, n_{3}\right)}$ и $C_{\mathrm{M}}\left(\alpha_{1}, \alpha_{2}, \alpha_{3}\right)$ тогда принимает вид

$$
C_{\left(m_{1}, n_{1}\right)\left(m_{2}, n_{2}\right)\left(m_{3}, n_{3}\right)}=f_{m_{1}, m_{2}, m_{2}} f_{n_{1}, n_{2}, n_{2}} C_{\mathrm{M}}\left(\alpha_{m_{1}, n_{1}}, \alpha_{m_{2}, n_{2}}, \alpha_{m_{3}, n_{3}}\right) .
$$

Очевидно, это "улучшение" теории не вполне согласуется с нашим стремлением к максимальной аналитичности. Однако на данный момент нам не известно, как можно обойтись без подобного наложения правил слияния "вручную".

3. Легко видеть, что выражения для центральных зарядов (7) и (25), как и для размерностей примарных полей (12) и (37), в теории поля Лиувилля и в ОММ просто связаны посредством аналитического продолжения параметров:

$$
b=-i \beta, \quad a=i \alpha, \quad Q=i q .
$$

Вместе с более аналитическими соотношениями, которые нам предстоит увидеть ниже, это может навести на мысль, что ОММ является просто аналитическим продолжением теории поля Лиувилля на чисто мнимые значения параметра $b$ (или наоборот). Мы вскоре увидим, что эта гипотеза несовместима с нашим непрерывным подходом. В частности, $C_{\mathrm{M}}\left(\alpha_{1}, \alpha_{2}, \alpha_{3}\right)$ не является аналитическим продолжением $C_{\mathrm{L}}\left(a_{1}, a_{2}, a_{3}\right)$. 


\section{4. КОНФОРМНЫЙ БУТСТРАП В ОММ}

Даже для формальных непрерывных полей $\Phi_{\alpha}$ условие отщепления нуль-векторов радикальньм образом влияет на вид операторного разложения. В частности, из уравнений (31) следует, что

$$
\begin{aligned}
& \Phi_{1,2} \Phi_{\alpha}=C_{+}^{(\mathrm{M})}(\alpha)\left[\Phi_{\alpha+\beta / 2}\right]+C_{-}^{(\mathrm{M})}(\alpha)\left[\Phi_{\alpha-\beta / 2}\right], \\
& \Phi_{2,1} \Phi_{\alpha}=\widetilde{C}_{+}^{(\mathrm{M})}(\alpha)\left[\Phi_{\alpha-\beta^{-1} / 2}\right]+\widetilde{C}_{-}^{(\mathrm{M})}(\alpha)\left[\Phi_{\alpha+\beta / 2}\right],
\end{aligned}
$$

где $C_{ \pm}^{(\mathrm{M})}(\alpha)=C_{\mathrm{M}}\left(\alpha_{1,2}, \alpha, \alpha \pm \beta / 2\right)$ и $\widetilde{C}_{ \pm}^{(\mathrm{M})}(\alpha)=C_{\mathrm{M}}\left(\alpha_{2,1}, \alpha, \alpha \mp \beta / 2\right)-$ специальные структурные константы, связанные с непрерывной функцией $C_{\mathrm{M}}\left(\alpha_{1}, \alpha_{2}, \alpha_{3}\right)$ посредством подходяшей специализации.

Далее мы следуем стандартному подходу конечномерного бутстрапа, развитому в статье [5] и многих последуюших работах. Вычисления, которые почти буквально следуют связанной с теорией Лиувилля работе Тешнера [9], приводят к двум функциональным соотношениям на $C_{\mathrm{M}}\left(\alpha_{1}, \alpha_{2}, \alpha_{3}\right)$ :

$$
\begin{aligned}
& \frac{C_{+}^{(\mathrm{M})}\left(\alpha_{1}\right) C_{\mathrm{M}}\left(\alpha_{1}+\beta / 2, \alpha_{2}, \alpha_{3}\right)}{C_{-}^{(\mathrm{M})}\left(\alpha_{1}\right) C_{\mathrm{M}}\left(\alpha_{1}-\beta / 2, \alpha_{2}, \alpha_{3}\right)}= \\
& =-\frac{\left(1-\beta^{2}-2 \alpha_{1} \beta\right)^{2} \gamma\left(\beta^{2} / 2+\left(\alpha_{3}+\alpha_{1}-\alpha_{2}\right) \beta\right)}{\gamma^{2}\left(\beta^{2}+2 \alpha_{1} \beta\right) \gamma\left(\beta^{2} / 2+\left(\alpha_{2}+\alpha_{3}-\alpha_{1}\right) \beta\right)} \times \\
& \quad \times \frac{\gamma\left(\beta^{2} / 2+\left(\alpha_{1}+\alpha_{2}-\alpha_{3}\right) \beta\right)}{\gamma\left(2-3 \beta^{2} / 2-\left(\alpha_{1}+\alpha_{2}+\alpha_{3}\right) \beta\right)}, \\
& \frac{\widetilde{C}_{+}^{(\mathrm{M})}\left(\alpha_{1}\right) C_{\mathrm{M}}\left(\alpha_{1}-\beta^{-1} / 2, \alpha_{2}, \alpha_{3}\right)}{\widetilde{C}_{-}^{(\mathrm{M})}\left(\alpha_{1}\right) C_{\mathrm{M}}\left(\alpha_{1}+\beta^{-1} / 2, \alpha_{2}, \alpha_{3}\right)}= \\
& =-\frac{\left(1-\beta^{-2}+2 \alpha_{1} \beta^{-1}\right)^{2} \gamma\left(\beta^{-2} / 2-\left(\alpha_{3}+\alpha_{1}-\alpha_{2}\right) \beta^{-1}\right)}{\gamma^{2}\left(\beta^{-2}-2 \alpha_{1} \beta^{-1}\right) \gamma\left(\beta^{-2} / 2-\left(\alpha_{2}+\alpha_{3}-\alpha_{1}\right) \beta^{-1}\right)} \times \\
& \quad \times \frac{\gamma\left(\beta^{-2} / 2-\left(\alpha_{1}+\alpha_{2}-\alpha_{3}\right) \beta^{-1}\right)}{\gamma\left(2-3 \beta^{-2} / 2+\left(\alpha_{1}+\alpha_{2}+\alpha_{3}\right) \beta^{-1}\right)} .
\end{aligned}
$$

Прежде всего используем эти уравнения для того, чтобы воспроизвести явные выражения для специальных структурных констант $C_{ \pm}^{(\mathrm{M})}(\alpha)$ и $\widetilde{C}_{ \pm}^{(\mathrm{M})}(\alpha)$. Возьмем $\alpha_{1}=\alpha_{3}=\alpha$ и $\alpha_{2}=\beta / 2$. Тогда с использованием соотношения (42) имеем

$$
\left(\frac{C_{+}^{(\mathrm{M})}(\alpha)}{C_{-}^{(\mathrm{M})}(\alpha)}\right)^{2}=\frac{\gamma(2 \alpha \beta) \gamma\left(2-\beta^{2}-2 \alpha \beta\right)}{\gamma\left(2-2 \beta^{2}-2 \alpha \beta\right) \gamma\left(\beta^{2}+2 \alpha \beta\right)} .
$$

Аналогично соотношение (43) дает

$$
\left(\frac{\widetilde{C}_{+}^{(\mathrm{M})}(\alpha)}{\widetilde{C}_{-}^{(\mathrm{M})}(\alpha)}\right)^{2}=\frac{\gamma\left(-2 \alpha \beta^{-1}\right) \gamma\left(2-\beta^{-2}+2 \alpha \beta^{-1}\right)}{\gamma\left(2-2 \beta^{-2}+2 \alpha \beta^{-1}\right) \gamma\left(\beta^{2}-2 \alpha \beta^{-1}\right)} .
$$


Подставляя (44) в (42), приходим к замкнутому функциональному соотношению для $C_{\mathrm{M}}\left(\alpha_{1}, \alpha_{2}, \alpha_{3}\right)$ :

$$
\begin{aligned}
& \frac{C_{\mathrm{M}}\left(\alpha_{1}+\beta, \alpha_{2}, \alpha_{3}\right)}{C_{\mathrm{M}}\left(\alpha_{1}, \alpha_{2}, \alpha_{3}\right)}=\frac{\gamma\left(\beta^{2}+\left(\alpha_{1}+\alpha_{2}-\alpha_{3}\right) \beta\right) \gamma\left(\beta^{2}+\left(\alpha_{3}+\alpha_{1}-\alpha_{2}\right) \beta\right)}{\gamma\left(\left(\alpha_{2}+\alpha_{3}-\alpha_{1}\right) \beta\right) \gamma\left(2-2 \beta^{2}-\left(\alpha_{1}+\alpha_{2}+\alpha_{3}\right) \beta\right)} \times \\
& \quad \times\left(\gamma\left(\beta^{2}+2 \alpha_{1} \beta\right) \gamma\left(2 \beta^{2}+2 \alpha_{1} \beta\right) \gamma\left(-1+2 \beta^{2}+2 \alpha_{1} \beta\right) \gamma\left(-1+3 \beta^{2}+2 \alpha_{1} \beta\right)\right)^{-1 / 2} .
\end{aligned}
$$

Второе функциональное соотношение, которое составляется из (43) и (45), отличается от первого простой подстановкой $\beta \rightarrow-\beta^{-1}$ :

$$
\begin{aligned}
& \frac{C_{\mathrm{M}}\left(\alpha_{1}-\beta^{-1}, \alpha_{2}, \alpha_{3}\right)}{C_{\mathrm{M}}\left(\alpha_{1}, \alpha_{2}, \alpha_{3}\right)}= \\
& =\frac{\gamma\left(\beta^{-2}-\left(\alpha_{1}+\alpha_{2}-\alpha_{3}\right) \beta^{-1}\right) \gamma\left(\beta^{-2}-\left(\alpha_{3}+\alpha_{1}-\alpha_{2}\right) \beta^{-1}\right)}{\gamma\left(-\left(\alpha_{2}+\alpha_{3}-\alpha_{1}\right) \beta^{-1}\right) \gamma\left(2-2 \beta^{-2}+\left(\alpha_{1}+\alpha_{2}+\alpha_{3}\right) \beta^{-1}\right)} \times \\
& \quad \times\left(\gamma\left(\beta^{-2}-2 \alpha_{1} \beta^{-1}\right) \gamma\left(2 \beta^{-2}-2 \alpha_{1} \beta^{-1}\right) \times\right. \\
& \left.\quad \times \gamma\left(2 \beta^{-2}-2 \alpha_{1} \beta^{-1}-1\right) \gamma\left(3 \beta^{-2}-2 \alpha_{1} \beta^{-1}-1\right)\right)^{-1 / 2} .
\end{aligned}
$$

Поскольку все приведенные бутстрапные вычисления являются явно аналитичными по $\beta$ и $\alpha$, не удивительно, что эти функциональные соотношения (с точностью до множителей, зависящих только от параметра $\alpha_{1}$, но не от комбинаций различных $\alpha$, а потому чувствительных к нормировке операторов) дают в точности аналитическое продолжение подобных же функциональных соотношений (21) в теории поля Лиувилля, когда

$$
b \rightarrow-i \beta, \quad b^{-1} \rightarrow i \beta, \quad a \rightarrow i \alpha .
$$

Однако по причинам, которые объясняются ниже, попытка построить вещественное решение $\beta$ с помощью такого продолжения терпит крах, и решение, представленное в следуюшем разделе, не является аналитическим продолжением лиувиллевской трехточечной функции (20).

\section{5. ТРЕХТОЧЕЧНАЯ ФУНКЦИЯ ОММ}

Для вешественных и несоизмеримых $\beta$ и $\beta^{-1}$ решение системы (46), (47) единственно. Его можно выразить в терминах той же самой специальной функции $\Upsilon(x)=\Upsilon_{\beta}(x)$, что и в уравнении (20), но на этот раз с параметром $\beta$ вместо $b$ :

$$
\begin{aligned}
C_{\mathrm{M}}\left(\alpha_{1}, \alpha_{2}, \alpha_{3}\right)= & \frac{A \Upsilon\left(\alpha_{1}+\alpha_{2}-\alpha_{3}+\beta\right) \Upsilon\left(\alpha_{2}+\alpha_{3}-\alpha_{1}+\beta\right)}{\left[\Upsilon\left(2 \alpha_{1}+\beta\right) \Upsilon\left(2 \alpha_{1}+2 \beta-\beta^{-1}\right) \Upsilon\left(2 \alpha_{2}+\beta\right)\right.} \times \\
& \times \frac{\Upsilon\left(\alpha_{3}+\alpha_{1}-\alpha_{2}+\beta\right) \Upsilon\left(2 \beta-\beta^{-1}+\alpha_{1}+\alpha_{2}+\alpha_{3}\right)}{\left.\Upsilon\left(2 \alpha_{2}+2 \beta-\beta^{-1}\right) \Upsilon\left(2 \alpha_{3}+\beta\right) \Upsilon\left(2 \alpha_{3}+2 \beta-\beta^{-1}\right)\right]^{1 / 2}}
\end{aligned}
$$

где нормировочный множитель

$$
A=\frac{\beta^{\beta^{-2}-\beta^{2}-1}\left[\gamma\left(\beta^{2}\right) \gamma\left(\beta^{-2}-1\right)\right]^{1 / 2}}{\Upsilon(\beta)}
$$


определяется из условия нормировки $C_{\mathrm{M}}(0, \alpha, \alpha)=1$ (мы настаиваем на интерпретации $\Phi_{0}$ как единичного оператора в ОММ).

В частности, усеченные операторные разложения (41) имеют явный вид

$$
\begin{aligned}
\Phi_{1,2} \Phi_{\alpha}= & {\left[\frac{\gamma\left(\beta^{2}\right) \gamma\left(2 \alpha \beta+2 \beta^{2}-1\right)}{\gamma\left(2 \beta^{2}-1\right) \gamma\left(\beta^{2}+2 \alpha \beta\right)}\right]^{1 / 2}\left[\Phi_{\alpha+\beta / 2}\right]+} \\
& +\left[\frac{\gamma\left(\beta^{2}\right) \gamma\left(2 \alpha \beta+\beta^{2}-1\right)}{\gamma\left(2 \beta^{2}-1\right) \gamma(2 \alpha \beta)}\right]^{1 / 2}\left[\Phi_{\alpha-\beta / 2}\right], \\
\Phi_{2,1} \Phi_{\alpha}= & {\left[\frac{\gamma\left(\beta^{-2}\right) \gamma\left(2 \beta^{-2}-2 \alpha \beta^{-1}-1\right)}{\gamma\left(2 \beta^{-2}-1\right) \gamma\left(\beta^{-2}-2 \alpha \beta^{-1}\right)}\right]^{1 / 2}\left[\Phi_{\alpha-\beta^{-1} / 2}\right]+} \\
& +\left[\frac{\gamma\left(\beta^{-2}\right) \gamma\left(\beta^{-2}-2 \alpha \beta^{-1}-1\right)}{\gamma\left(2 \beta^{-2}-1\right) \gamma\left(-2 \alpha \beta^{-1}\right)}\right]^{1 / 2}\left[\Phi_{\alpha+\beta / 2}\right] .
\end{aligned}
$$

Мы проверили на многих частных примерах, что, коль скоро выражение (49) взято при допустимых вырожденных значениях $\alpha_{i}=\alpha_{m_{i}, n_{i}}$, оно редуцируется в точности к произведению гамма-функций, как у Доценко и Фатеева. Важно, что набор из трех $\alpha_{m_{i}, n_{i}}$ в структурной константе удовлетворяет правилам слияния (35). Иначе выражение (49) не всегда обеспечивает обрашение структурной константы в нуль. Часто оно дает некоторые конечные числа, смысл и возможная интерпретация которых остаются для нас загадкой.

Было бы очень важно в обшем случае доказать, что для допустимых вырожденных $\alpha_{i}$ выражение (49) и формулы Доценко-Фатеева совпадают.

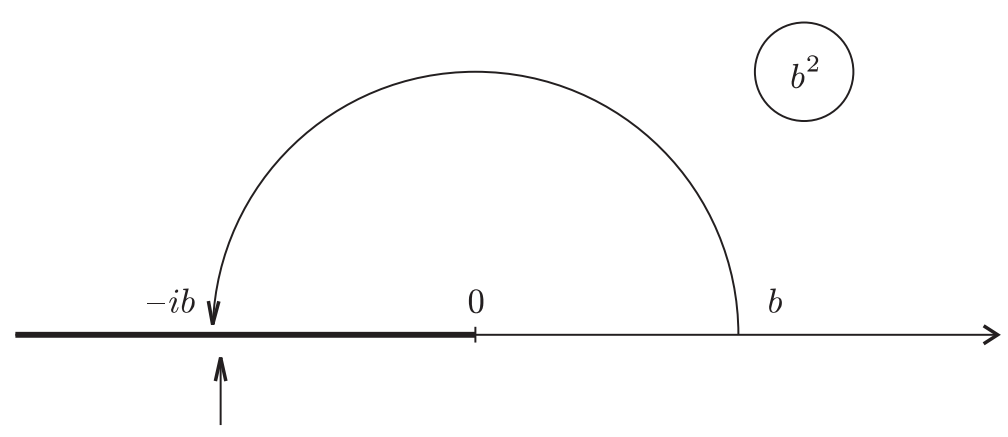

Естественная граница аналитичности $\Upsilon_{b}$

Рис. 1

Решение (49) не есть аналитическое продолжение лиувиллевской трехточечной функции $(20)$. Дело в том, что функция $\Upsilon_{b}(x)$ как функция параметра $b$ аналитична на всей комплексной плоскости $b^{2}$, за исключением отрицательной части вешественной оси, где она встречает естественную гранищу аналитичности (см. рис. 1). В следуюшем разделе мы несколько подробнее остановимся на вопросе аналитического соотношения между решениями (20) и (49). 


\section{6. АНАЛИТИЧЕСКОЕ ПРОДОЛЖЕНИЕ ПО $b^{2}$}

Как отмечалось в предыдушем разделе, $C_{\mathrm{L}}\left(a_{1}, a_{2}, a_{3} \mid b\right)$ не может быть аналитически продолжена на чисто мнимые значения параметра $b$. По той же самой причине $C_{\mathrm{M}}\left(\alpha_{1}\right.$, $\left.\alpha_{2}, \alpha_{3} \mid \beta\right)$ не допускает аналитического продолжения на чисто мнимые значения $\beta$. В то же время $C_{\mathrm{L}}\left(a_{1}, a_{2}, a_{3} \mid b\right)$ и $C_{\mathrm{M}}\left(-i a_{1},-i a_{2},-i a_{3} \mid i b\right)$ удовлетворяют одному и тому же набору соотношений функционального сдвига (с точностью до некоторых зависящих от нормировки множителей), что позволяет единственным образом восстановить $C_{\mathrm{M}}\left(-i a_{1},-i a_{2},-i a_{3} \mid i b\right)$ или $C_{\mathrm{L}}\left(a_{1}, a_{2}, a_{3} \mid b\right)$ в любом положительном иррациональном или отришательном иррациональном значении $b^{2}$, соответственно. Для прояснения аналитического соотношения между этими двумя функциями сравним их в тех комплексных значениях $b^{2}$, где они обе являются корректно определенными. При комплексных значениях $b$ соотношения сдвига определяют решение только с точностью до эллиптической функции с периодами $b$ и $b^{-1}$. Возьмем функцию $C_{\mathrm{L}}\left(a_{1}, a_{2}, a_{3} \mid b\right)$ при некотором комплексном значении $b^{2}$ в нижней полуплоскости, как показано на рис. 2 , таким образом, чтобы ее можно было без опаски аналитически продолжить в точку $\beta=i b$ и сравнить с $C_{\mathrm{M}}\left(-i a_{1},-i a_{2},-i a_{3} \mid i b\right)$.

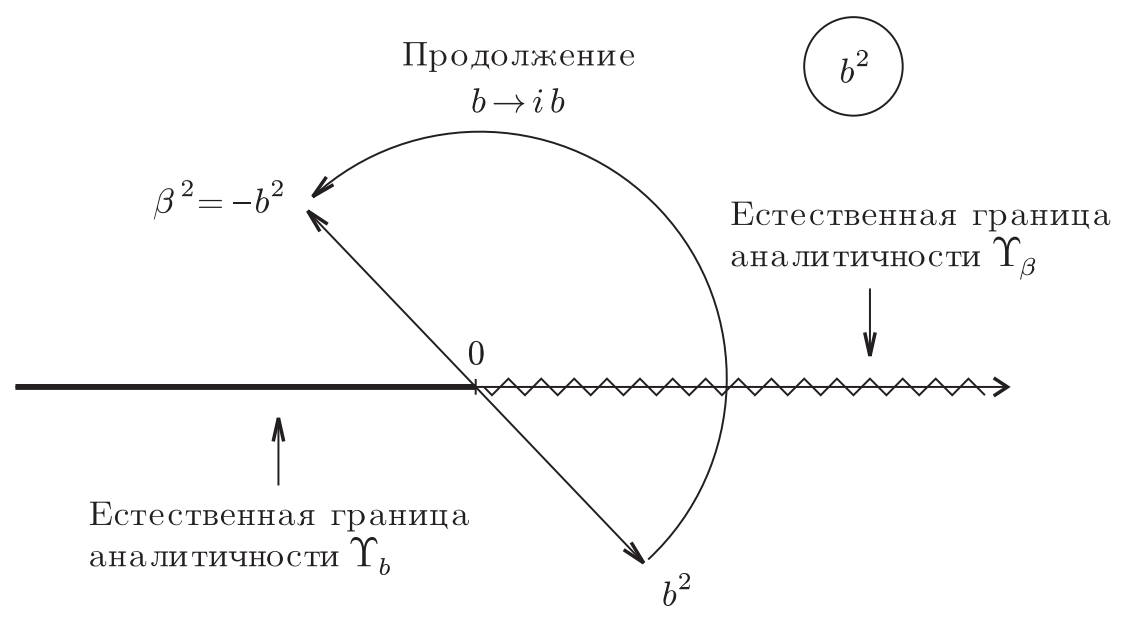

Рис. 2

Рассмотрим отношение

$$
R\left(a_{1}, a_{2}, a_{3} \mid b\right)=\frac{C_{\mathrm{M}}\left(-i a_{1},-i a_{2},-i a_{3} \mid i b\right)}{C_{\mathrm{L}}\left(a_{1}, a_{2}, a_{3} \mid b\right)} .
$$

В нем $\Upsilon$-функции из трехточечных функций ОММ и Лиувилля комбинируются в $\theta$-функции в силу следуюшего тождества (которое можно доказать непосредственно, что будет сделано в другом месте):

$$
\Upsilon_{b}(x) \Upsilon_{i b}(-i x+i b)=e^{i \pi\left(b-b^{-1}-2 x\right)^{2} / 8} \frac{\theta_{1}\left(\pi x b^{-1} \mid h\right)}{h^{1 / 4} \theta_{3}(0 \mid h)}
$$


где $\theta_{1}(x \mid h)$ и $\theta_{3}(x \mid h)$ - обычные эллиптические $\theta$-функции и $h=e^{i \pi b^{-2}}$. Подразумевается, что $b^{2}$ в уравнении (53) имеет отрицательную мнимую часть, так что $\Upsilon_{b}$ можно без опаски продолжить до $\Upsilon_{i b}$, обходя отрицательную часть вещественной оси $b^{2}$, как показано на рис. 2. Результат имеет вид

$$
R\left(a_{1}, a_{2}, a_{3}\right)=\frac{\left(\pi \mu \gamma\left(b^{2}\right)\right)^{\left(a_{1}+a_{2}+a_{3}-Q\right) / b}\left[\gamma\left(-b^{2}-1\right) \gamma\left(-b^{-2}\right)\right]^{1 / 2}}{b \prod_{i=1}^{3}\left[\gamma\left(2 a_{i} b-b^{2}-1\right) \gamma\left(2 a_{i} b^{-1}-b^{-2}\right)\right]^{1 / 2}} T\left(a_{1}, a_{2}, a_{3}\right)
$$

где

$$
\begin{aligned}
T\left(a_{1}, a_{2}, a_{3}\right)= & \frac{\theta_{1}\left(\pi b^{-1}\left(a_{1}+a_{2}-a_{3}\right)\right) \theta_{1}\left(\pi b^{-1}\left(a_{2}+a_{3}-a_{1}\right)\right)}{\pi \theta_{1}^{\prime}(0) \theta_{1}\left(2 \pi b^{-1} a_{1}\right) \theta_{1}\left(2 \pi b^{-1} a_{2}\right) \theta_{1}\left(2 \pi b^{-1} a_{3}\right)} \times \\
& \times \theta_{1}\left(\pi b^{-1}\left(a_{3}+a_{1}-a_{2}\right)\right) \theta_{1}\left(\pi b^{-1}\left(a_{1}+a_{2}+a_{3}\right)\right)
\end{aligned}
$$

(причем все $\theta$-функции имеют один и тот же модуль $h$ ) является, как легко проверить, эллиптической функцией по всем трем $a_{i}$ с периодами $b$ и $b^{-1}$. Таким образом, для комплексных $b^{2}$ два решения $C_{\mathrm{L}}\left(a_{1}, a_{2}, a_{3} \mid b\right)$ или $C_{\mathrm{M}}\left(-i a_{1},-i a_{2},-i a_{3} \mid i b\right)$ по сути одних и тех же функциональных соотношений различаются, как и следует, на дважды периодическую функцию (с точностью до “концевого” множителя в (54), который в действительности появляется из-за различных нормировок примарных полей в ОММ и теории поля Лиувилля).

\section{7. МИНИМАЛЬНАЯ ГРАВИТАЦИЯ}

Обратимся теперь к МГ, индуцированной ОММ $\mathcal{M}_{\beta}$ с полным центральным зарядом материи (25). Как широко известно, баланс центрального заряда (10) требует, чтобы параметр Лиувилля $b$ был равен ОММ-параметру $\beta$ (разумеется, есть и другое решение $b=\beta^{-1} ;$ мы выбираем первое с тем, чтобы сохранить условие $\left.b<1\right)$. Поэтому начиная с этого момента мы используем единое обозначение $b$ для этого параметра как в теории Лиувилля, так и в ОММ, подставляя

$$
\beta=b
$$

во все выражения, относящиеся к ОММ. Более того, “параметр одевания" а в уравнении (11) в МГ просто связан с параметром $\alpha$ примарного поля материи:

$$
a=\alpha+b
$$

Снова имеется вторая возможность $-a=b^{-1}-\alpha$, но такой выбор не дает ничего нового, поскольку аналитические выражения для корреляционных функций локальной теории поля позволяют отождествить эти два возможных одевания с точностью до нормировки [7]

$$
e^{2 a \phi}=D_{\mathrm{L}}(a) e^{2(Q-a) \phi},
$$


где $D_{\mathrm{L}}(a)$ взято из уравнения (24). Мы обозначим соответствуюший (формальный при общих $\alpha$ ) одетый оператор как

$$
U_{a}=\Phi_{a-b} e^{2 a \phi} .
$$

Трехточечная функция в МГ имеет простой вид:

$$
C^{(\mathrm{MG})}\left(a_{1}, a_{2}, a_{3}\right)=\left\langle C \bar{C}\left(x_{1}\right) \ldots C C\left(x_{3}\right)\right\rangle_{\mathrm{gh}}\left\langle U_{a_{1}}\left(x_{1}\right) U_{a_{2}}\left(x_{2}\right) U_{a_{3}}\left(x_{3}\right)\right\rangle_{\mathrm{MG}} .
$$

Координатная зависимость в правой части сокрашается и, как и ожидалось, мы получаем трехточечное корреляшионное число в МГ:

$$
C^{(\mathrm{MG})}\left(a_{1}, a_{2}, a_{3}\right)=C_{\mathrm{M}}\left(a_{1}-b, a_{2}-b, a_{3}-b \mid b\right) C_{\mathrm{L}}\left(a_{1}, a_{2}, a_{3} \mid b\right) .
$$

В этом произведении все $\Upsilon$-функции, зависяшие от комбинаций параметров $a_{i}$, сокращаются и остается (с точностью до общего множителя) произведение множителей, зависяших от каждого отдельного параметра $a_{i}$ (иногда называемых "концевыми" множителями):

$$
C^{(\mathrm{MG})}\left(a_{1}, a_{2}, a_{3}\right)=\left(\pi \mu \gamma\left(b^{2}\right)\right)^{\left(Q-\sum_{i=1}^{3} a_{i}\right) / b}\left[\frac{\gamma\left(b^{2}\right) \gamma\left(b^{-2}-1\right)}{b^{2}}\right]^{1 / 2} \prod_{i=1}^{3} F_{\mathrm{MG}}\left(a_{i}\right),
$$

где выбрана следуюшая нормировка концевых множителей:

$$
F_{\mathrm{MG}}(a)=\left[\gamma\left(2 a b-b^{2}\right) \gamma\left(2 a b^{-1}-b^{-2}\right)\right]^{1 / 2} .
$$

Эта факторизованная форма трехточечной функции в МГ была ранее (уже давно) установлена в подходе матричных моделей [12], равно как и в рамках несколько иного подхода к теории поля Лиувилля [13].

Разумеется, с технической стороны гораздо легче не решать функциональные соотношения (21) и (46), (47) по отдельности для каждой из корреляционных функций Лиувилля и ОММ, а скомбинировать их в функциональные соотношения для произведения. Таким путем полностью исключается всякая потребность в сложных специальных функциях $\Upsilon$. Комбинируя (21) и (46), (47) и учитывая соотношение (57), находим уравнения

$$
\begin{gathered}
\frac{C_{\mathrm{MG}}\left(a_{1}+b, a_{2}, a_{3}\right)}{C_{\mathrm{MG}}\left(a_{1}, a_{2}, a_{3}\right)}=\frac{\left(2 a_{1} b^{-1}-b^{-2}\right)\left(2 a_{1} b^{-1}+1-b^{-2}\right)}{\pi \mu \gamma\left(b^{2}\right)}\left(\frac{\gamma\left(2 a_{1} b+b^{2}\right)}{\gamma\left(2 a_{1} b-b^{2}\right)}\right)^{1 / 2}, \\
\frac{C_{\mathrm{MG}}\left(a_{1}+b^{-1}, a_{2}, a_{3}\right)}{C_{\mathrm{MG}}\left(a_{1}, a_{2}, a_{3}\right)}=\frac{\left(2 a_{1} b-b^{2}+1\right)\left(2 a_{1} b-b^{2}\right)}{\pi \tilde{\mu} \gamma\left(b^{-2}\right)}\left(\frac{\gamma\left(2 a_{1} b^{-1}+b^{-2}\right)}{\gamma\left(2 a_{1} b^{-1}-b^{-2}\right)}\right)^{1 / 2} .
\end{gathered}
$$

Этого достаточно для восстановления факторизованной формы (62) с точностью до не зависяшей от $\alpha$ обшей нормировки. В данной работе мы выбрали более сложную схему 
отдельного вычисления ОММ-структурной функции, с тем чтобы получить больше сведений об отдельных вкладах лиувиллевских и ОММ-степеней свободы в результат (62) для МГ.

Наконец, нам нужно получить аналитическое выражение при вырожденных значениях параметров поля материи. На этом шаге следует добавить множитель $f_{m_{1}, m_{2}, m_{2}} \times$ $f_{n_{1}, n_{2}, n_{2}}$ для обеспечения правил слияния в случае вырожденных ОММ-полей. Получаем следуюшее выражение для трехточечных корреляционных чисел МГ:

$$
\begin{aligned}
C_{\left(m_{1}, n_{1}\right)\left(m_{2}, n_{2}\right)\left(m_{3}, n_{3}\right)}^{(\mathrm{MG})}= & f_{m_{1}, m_{2}, m_{2}} f_{n_{1}, n_{2}, n_{2}}\left(\pi \mu \gamma\left(b^{2}\right)\right)^{\left(Q-\sum_{i=1}^{3} a_{m_{i}, n_{i}}\right) / b} \times \\
& \times\left[\frac{\gamma\left(b^{2}\right) \gamma\left(b^{-2}-1\right)}{b^{2}}\right]^{1 / 2} \prod_{i=1}^{3} F_{\mathrm{MG}}\left(a_{m_{i}, n_{i}}\right) .
\end{aligned}
$$

Как отмечалось выше, лиувиллевская трехточечная функция не нормирована. Чтобы получить нормированное выражение, по-прежнему следует разделить ее на лиувиллевскую статистическую сумму. Это будет сделано в следуюшем разделе.

\section{8. ДВУХТОЧЕЧНАЯ ФУНКЦИЯ И НОРМИРОВКА}

Непосредственное вычисление двухточечных корреляционных чисел, в отличие от трехточечных, не является простой задачей. При прямом вычислении следует отдельно рассмотреть задачу о фиксашии остающейся калибровочной симметрии на сфере. Для того чтобы избежать этой сложной задачи, мы предпочитаем воспользоваться тождеством

$$
-\frac{\partial}{\partial \mu}\left\langle U_{a} U_{a}\right\rangle_{\mathrm{g}}=\left\langle U_{a} U_{a} U_{b}\right\rangle_{\mathrm{g}}
$$

которое непосредственно следует из действия (9) и лагранжиана Лиувилля (6). Это дает следуюшее выражение для (ненормированного) двухточечного корреляционного числа в МГ:

$$
\left\langle U_{a} U_{a}\right\rangle_{\mathrm{MG}}=\left(\pi \mu \gamma\left(b^{2}\right)\right)^{(Q-2 a) / b} \frac{F_{\mathrm{MG}}^{2}(a)}{\pi(2 a-Q)} .
$$

Аналогичное соотношение

$$
-\frac{\partial^{3}}{\partial \mu^{3}} Z_{\mathrm{L}}=C_{\mathrm{MG}}(b, b, b)=\left(\pi \mu \gamma\left(b^{2}\right)\right)^{Q / b} \frac{\gamma\left(2-b^{-2}\right)}{\pi^{3} \gamma\left(b^{2}\right) \mu^{3} b}
$$

можно использовать для восстановления лиувиллевской статистической суммы [6]:

$$
Z_{\mathrm{L}}=\left(\pi \mu \gamma\left(b^{2}\right)\right)^{Q / b} \frac{\left(1-b^{2}\right)}{\pi^{3} \gamma\left(b^{2}\right) \gamma\left(b^{-2}\right) Q} .
$$


С учетом этого выражения нормированные двух- и трехточечные корреляционные числа в ОММ имеют вид

$$
\begin{aligned}
\frac{\left\langle U_{a} U_{a}\right\rangle_{\mathrm{MG}}}{Z_{\mathrm{L}}}= & \frac{\left(\pi \mu \gamma\left(b^{2}\right)\right)^{-2 a / b} \pi^{2} \gamma\left(b^{2}\right) \gamma\left(b^{-2}\right) Q}{\left(1-b^{2}\right)(2 a-Q)} F_{\mathrm{MG}}^{2}(a), \\
\frac{C^{(\mathrm{MG})}\left(a_{1}, a_{2}, a_{3}\right)}{Z_{\mathrm{L}}}= & \left(\pi \mu \gamma\left(b^{2}\right)\right)^{-\sum_{i=1}^{3} a_{i} / b} \times \\
& \times \frac{\pi^{3}\left(1+b^{2}\right)\left[-\gamma^{3}\left(b^{2}\right) \gamma^{3}\left(b^{-2}\right)\right]^{1 / 2}}{\left(1-b^{2}\right)^{2}} \prod_{i=1}^{3} F_{\mathrm{MG}}\left(a_{i}\right) .
\end{aligned}
$$

Выражения (70) и (71) вместе с представлением (49) для структурных констант в ОММ являются основным результатом данной работы.

Благодарности. Эта работа - для Галины Гриценко. Что еще я мог бы для нее сделать? Я также благодарен А. Замолодчикову и И. Костову за полезные обсуждения. Работа была поддержана со стороны European Committee, contract HPRN-CT-200200325

\section{Список литературы}

[1] A. Polyakov. Phys. Lett. B. 1981. V. 103. P. 207.

[2] J. Distler, H. Kawai. Nucl. Phys. B. 1989. V. 231. P. 509; F. David. Mod. Phys. Lett. A. 1988. V. 3. P. 1651.

[3] I. R. Klebanov. String theory in two dimensions. hep-th/9108019; P. Ginsparg, G. Moore. Lectures on 2D gravity and 2D string theory. TASI summer school, 1992; hep-th/9304011; P. Di Francesco, P. Ginsparg, J. Zinn-Justin. Phys. Rep. 1995. V. 254. P. 1.

[4] V. Knizhnik, A. Polyakov, A. Zamolodchikov. Mod. Phys. Lett. A. 1988. V. 3. P. 819.

[5] A. Belavin, A. Polyakov, A. Zamolodchikov. Nucl. Phys. B. 1984. V. 241. P. 333.

[6] H. Dorn, H.-J. Otto. Phys. Lett. B. 1992. V. 291. P. 39; hep-th/9206053; Nucl. Phys. B. 1994. V. 429. P. 375; hep-th/9403141.

[7] A. B. Zamolodchikov, Al. B. Zamolodchikov. Nucl. Phys. B. 1996. V. 477. P. 577; hep-th/9506136.

[8] E. W. Barnes. Proc. London. Math. Soc. 1899. V. 31. P. 358; Phil. Trans. Roy. Soc. A. 1901. V. 196. P. 265.

[9] J. Teschner. Phys. Lett. B. 1995. V. 363. P. 65; hep-th/9507109.

[10] V. G. Kac. Infinite Dimensional Lie Algebras. An Introduction. Progr. Math. V. 44. Boston, MA: Birkhäuser, 1983.

[11] V. Dotsenko, V. Fateev. Nucl. Phys. B. 1985. V. 251. P. 691; Phys. Lett. B. 1985. V. 154. P. 291.

[12] P. Di Francesco, D. Kutasov. Nucl. Phys. B. 1990. V. 342. P. 589.

[13] M. Goulian, M. Li. Phys. Rev. Lett. B. 1991. V. 264. P. 292. 